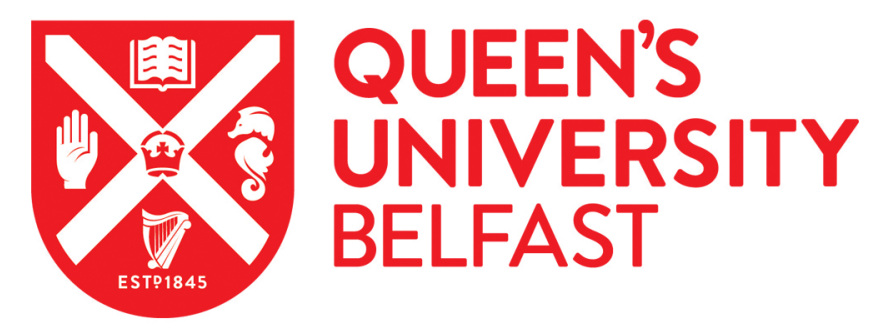

\title{
Protection of DNA against low-energy electrons by amino acids: A first-principles molecular dynamics study
}

Gu, B., Smyth, M., \& Kohanoff, J. (2014). Protection of DNA against low-energy electrons by amino acids: A first-principles molecular dynamics study. Physical Chemistry Chemical Physics , 16(44), 24350-24358. https://doi.org/10.1039/c4cp03906h

\section{Published in:}

Physical Chemistry Chemical Physics

\section{Document Version:}

Peer reviewed version

Queen's University Belfast - Research Portal:

Link to publication record in Queen's University Belfast Research Portal

\section{General rights}

Copyright for the publications made accessible via the Queen's University Belfast Research Portal is retained by the author(s) and / or other copyright owners and it is a condition of accessing these publications that users recognise and abide by the legal requirements associated with these rights.

Take down policy

The Research Portal is Queen's institutional repository that provides access to Queen's research output. Every effort has been made to ensure that content in the Research Portal does not infringe any person's rights, or applicable UK laws. If you discover content in the Research Portal that you believe breaches copyright or violates any law, please contact openaccess@qub.ac.uk. 


\title{
Protection of DNA against low-energy electrons by amino
}

\section{acids: a first-principles molecular dynamics study}

\author{
Bin Gu ${ }^{a, b}$, Maeve $\operatorname{Smyth}^{b}$, and Jorge Kohanoff ${ }^{b, *}$
}

\author{
Received Xth XXXX 2014, Accepted Xth XXXX $201 X$
}

First published on the web Xth XXXX $201 X$

DOI: 10.1039/b000000x

Using first-principles molecular dynamics simulations, we have investigated the notion that amino acids can play a protective role when DNA is exposed to excess electrons produced by ionizing radiation. In this study we focus on the interaction of glycine with the DNA nucleobase thymine. We studied thymineglycine dimers and a condensed phase model consisting of one thymine molecule solvated in amorphous glycine. Our results show that the amino acid acts as a protective agent for the nucleobase in two ways. If the excess electron is initially captured by the thymine, then a proton is transferred in a barrier-less way from a neighboring hydrogen-bonded glycine. This stabilizes the excess electron by reducing the net partial charge on the thymine. In the second mechanism the excess electron is captured by a glycine, which acts as a electron scavenger that prevents electron localization in DNA. Both these mechanisms introduce obstacles to further reactions of the excess electron within a DNA strand, e.g. by raising the free energy barrier associated with strand breaks.

\footnotetext{
${ }^{a}$ Department of Physics, Nanjing University of Information Science and Technology, Nanjing 210044, China

${ }^{b}$ Atomistic Simulation Centre, Queen's University Belfast, Belfast BT7 1NN, Northern Ireland, UK

${ }^{*}$ E-mail:j.kohanoff@qub.ac.uk.
} 


\section{Introduction}

Low energy electrons (LEEs) with energy bellow $20 \mathrm{eV}$ are the most abundant secondary products along energy depositing tracks of ionizing radiation through a biological medium ${ }^{1-3}$. The effect of these low energy electrons on the components of DNA has attracted attention for over a decade. Theoretical studies have mostly focused on the interaction of an excess electron with DNA components in the gas phase, beginning with isolated nucleobases ${ }^{4-6}$ and extending to large systems including dinucleoside phosphate deoxyguanylyl-3',5'-deoxycytidine dimer $\left[\mathrm{dGpdC}_{2}{ }^{7,8}\right.$. In addition, the effect of the surrounding medium has been considered through microsolvation ${ }^{9,10}$, polarised continuum model $(\mathrm{PCM})^{11}$, and more recently through condensed phase simulations ${ }^{12}$. Experiments have been carried out on the components of DNA, on dry plasmid DNA and with thin layers of water ${ }^{13}$. However, in the cellular environment, DNA is always bound to other molecules such as proteins and solvated ions ${ }^{14}$. As a first step to representing the physiological environment and understanding the irradiation response of living cells under realistic conditions, it is important to study the influence of neighboring amino acids.

There have been both experimental and theoretical investigations focusing on the interactions of amino acids with DNA components, including not only the traditionally categorized general hydrogen bonding $^{15,16}$, ionic and van der Waals interactions ${ }^{14,17}$, but also the specific stacking and T-shaped interactions between DNA nucleobases and amino acids with aromatic side chains ${ }^{18-20}$. Gas-phase studies have highlighted that a barrier-free proton transfer occurs between anionic nucleobases and amino acids or between nucleobases ${ }^{21-23}$. Indeed, this important interaction provides a base-recognition technique by certain proteins $15,24,25$. Hydrogen bonds, in turn, have been shown to have a stabilising effect on anionic nucleobase structures. This was shown through microsolvation and condensed-phase studies ${ }^{12,26}$, which highlighted proton acceptor sites as particularly able to stablise a structure.

Recently, Solomun et al reported that the single strand DNA-binding $E$. coli protein can effectively inhibit single strand breaks (SSB) of oligonucleotides induced by 3 eV LEEs $^{27}$. Furthermore, Ptasińska et al reported the fragmentation of short DNA strands irradiated by a $1 \mathrm{eV}$ electron beam in the presence of the amino acids glycine and arginine ${ }^{28}$. Their results show that DNA damage is promoted at low amino 

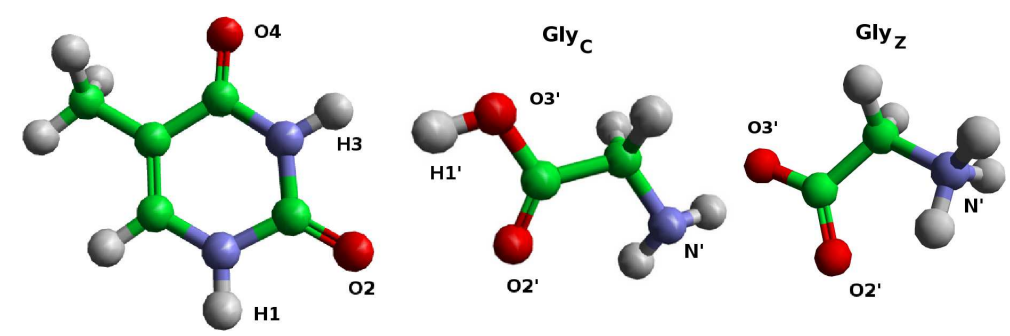

Fig. 1 (colour online) The structures of thymine (left), canonical (middle) and zwitterionic (right) glycine molecules are shown. The relevant atoms involved in the hydrogen bonds in T-Gly dimers have been labeled.

acid concentrations. However, when the ratio between amino acids and oligonucleotides is larger than 0.5 , the observed damage decreases with increasing ratio. It has been suggested that there are two underlying mechanisms influencing these findings. Firstly the protein may create a physical shielding to the DNA. In addition the bonding interactions may play an essential role in quenching SSB through the stabilization of the anionic species. In order to understand these processes the basic question is: how do low energy electrons interact with a mixture of amino acids and DNA components? The elucidation of these mechanisms at the atomic scale under realistic conditions is important to further our understanding of radiation effects in biological systems.

Therefore, in this study we examined the interactions between thymine, which has the highest electron affinity amongst the four nucleobases in DNA ${ }^{12,29}$, and glycine. Glycine was chosen as it is non-polar and has the shortest side chain among standard amino acids. It is a simple model which can emulate the properties of common non-polar amino acids. This choice was also dictated by the availability of experimental results ${ }^{28}$. When polar amino acids are involved, the hydrogen-bonding possibilities will be increased ${ }^{17}$. Therefore, the hydrogen-bonding related protection to DNA by the none-polar glycine might take place naturally by those polar amino acids, such as arginine, as also shown in experiments of Ptasińska et $_{\mathbf{a l}} \mathbf{2}^{28}$. The molecular structures of thymine and glycine are shown in Fig. 1. In our simulations, the LEEs was represented by the vertically attached excess electrons with zero kinetic energy. 


\section{Methods and simulation details}

We began by investigating the interaction of a thymine with a single glycine forming three geometrically different isolated dimers (T-Gly), which mimic the 1:1 ratio between amino acids and nucleobases. For the situations of high ratio of amino acids, we created two liquid phase configurations consisting of a single thymine solvated by 32 glycines $\left(\mathrm{T}_{-} \mathrm{Gly}_{32}\right)$, under periodic boundary conditions. In these initial configurations the thymine was coordinated to one glycine as in the isolated T-Gly dimers.

The starting molecular structure and system configurations were obtained using the molecular configuration editor Aten ${ }^{30}$. The starting configurations for the first-principles simulations were taken from snapshots of classical molecular dynamics (MD) simulations using the CHARMM force fields ${ }^{31,32}$ implemented in the DL_Poly package ${ }^{33}$.

The ab initio calculations were carried out using the quantum module Quickstep (QS) from the open source code $\mathrm{CP} 2 \mathrm{~K}^{34}$. The calculations were performed at the DFT level of theory using the Gaussian and augmented plane waves method (GAPW), in which the electrostatic energy and potential are calculated by Fourier transform techniques and the Kohn-Sham orbitals are expanded on a Gaussian basis set ${ }^{\mathbf{3 5}}$.

For isolated T-Gly dimers, we applied the Martyna Tuckerman method for the Poisson solver, which decouples periodic images of the system ${ }^{36}$. These calculations were carried out at the all-electron hybrid PBE0 ${ }^{37}$ level of theory, with the $6-311++\mathrm{G}^{* *}$ basis which includes diffuse orbitals on all atoms. The quality of the $\mathrm{PBE}^{38}$ pure DFT functional used during condensed phase calculations was also assessed against PBE0. For condensed state simulations, we applied periodic boundary conditions to the Poisson solver. Goedecker-Teter-Hutter pseudopotentials ${ }^{39}$, a TZVP-GTH basis set, and the PBE $^{38}$ exchange-correlation functional were utilized. All simulations were carried out with a time step of $1 \mathrm{fs}$.

In all cases, the excess electron was attached to the LUMO of the system. After that, a geometry optimization was carried out to relax the negatively charged system. A compensating uniform background was automatically added in order to keep the system formally neutral. 


\section{Results and discussion}

\subsection{Thymine-glycine dimers}

Out of a variety of T-Gly dimers, we selected the three exhibiting the lowest energy. Their optimized neutral structures by the conjugated gradient (CG) method at the level of PBE0 theory, are depicted in left panel of Fig. 2. The dotted lines represent hydrogen bonds between thymine and glycine. Each dimer exhibits two inter-molecular hydrogen bonds. For convenience, we have named each dimer as follows: $\mathrm{T}_{H_{1}-O_{2}^{\prime}}^{O_{2}-H_{1}^{\prime}}$ Gly as $\mathrm{T}_{1,2}$-Gly, $\mathrm{T}_{H_{3}-O_{2}^{\prime}}^{O_{2}-H_{1}^{\prime}}$ Gly as $\mathrm{T}_{2,3}$-Gly, and $\mathrm{T}_{H_{3}-O_{2}^{\prime}}^{O_{4}-H_{1}^{\prime}}$ Gly as $\mathrm{T}_{3,4}$-Gly.
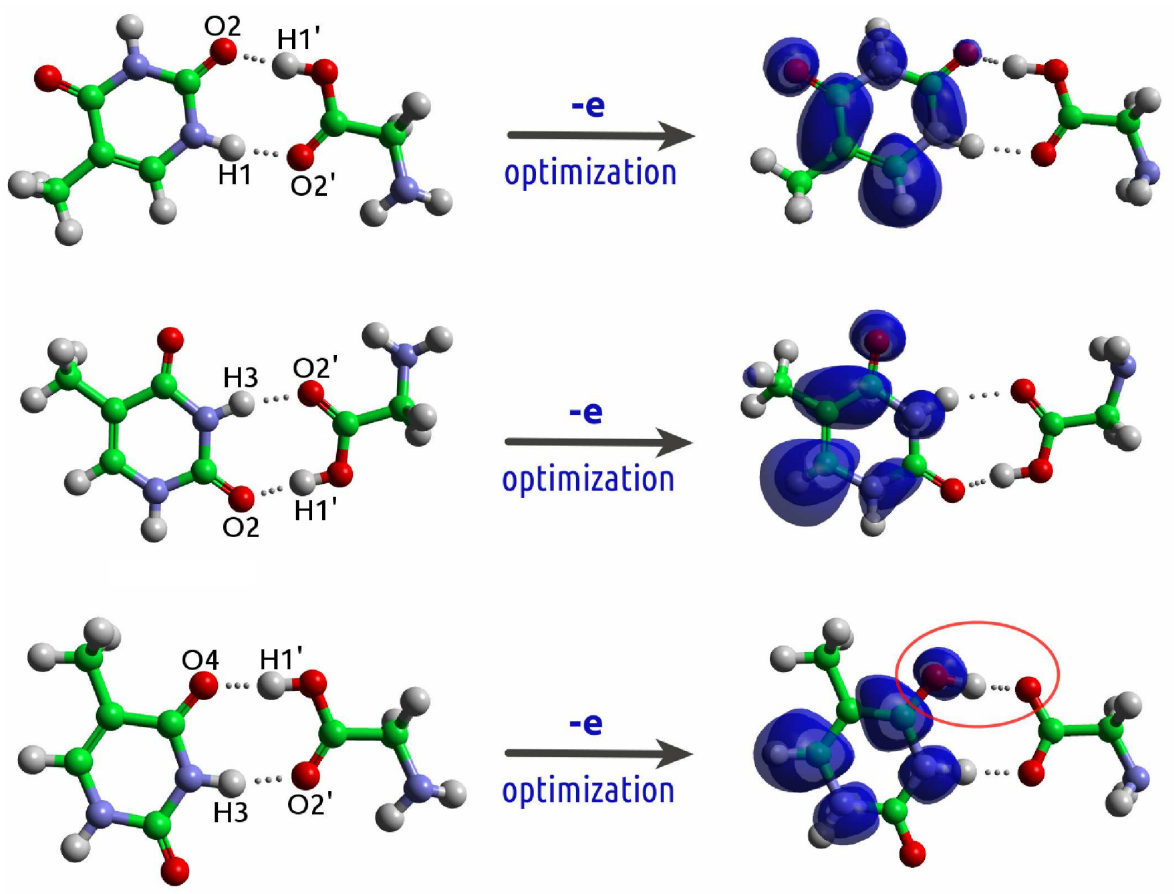

Fig. 2 (colour online) The optimized neutral T-Gly dimers (left) and the spin density distribution on their optimized anionic states with one excess electron (right). The hydrogen bonds were shown by dotted lines. From top to bottom: $\mathrm{T}_{1,2}$-Gly, $\mathrm{T}_{2,3}$-Gly and $\mathrm{T}_{3,4}$-Gly. The proton transfer in $\mathrm{T}_{3,4}$-Gly is highlighted with red circle.

\subsubsection{Structure}

The optimized structures of the anionic dimers by the CG method with PBE0 theory, and the spin density distributions on them are shown in the right panel of Fig. 2. It can be seen that the excess electron always occupies a valence bound state localized in the thymine. Hence, for all the three dimers, the total spin 
associated to the thymine, $\sigma_{T}$, is 1 , while the spin associated to the glycine, $\sigma_{G l y}$, is zero. This can be taken as an indication that the excess electron always localizes in the thymine end of the dimer.

Table 1 Adiabatic electron affinity (AEA) of thymine-glycine dimers, total atomic charges on the thymine $\left(q_{T}\right)$, and transfer strength of the $\mathrm{H}_{1}^{\prime}$ from glycine to thymine $\left(\xi_{H_{1}^{\prime} \rightarrow T}\right)$.

\begin{tabular}{cccc}
\hline Configuration & AEA $(\mathrm{eV})$ & $q_{T}(\mathrm{e})$ & $\xi_{H_{1}^{\prime} \rightarrow T}$ (a.u.) \\
\hline $\mathrm{T}_{1,2}$-Gly & 0.526 & -0.93 & -0.12 \\
$\mathrm{~T}_{2,3}$-Gly & 0.242 & -0.94 & -0.16 \\
$\mathrm{~T}_{3,4}$-Gly & 0.613 & -0.63 & 0.18 \\
\hline
\end{tabular}

The adiabatic electron affinity (AEA), defined as the difference between the total energies $\left(E_{t o t}\right)$ of the optimized ground states of $\mathrm{X}$ and its negative ion $\mathrm{X}^{-}: A E A=E_{t o t}\left(X^{-}\right)-E_{t o t}(X)$, was calculated for thymine, glycine and dimers. The results are listed in Table 1, along with the sum of partial atomic charges on the thymine component $\left(q_{T}\right)$. The partial atomic charges were calculated according to the Mulliken population analysis 40 .

For the isolated glycine the excess electron is not stable, and this is reflected in a negative and meaningless AEA. In the case of thymine, the AEA is only slightly negative, and it has been shown that the anion is stabilized by zero-point-motion (not included here) ${ }^{12}$. This very fact suggests that, in the dimers, the excess electron will prefer to localize on the thymine rather than on the glycine. The AEA of the dimers is positive, meaning that the anionic state is stable. This is due to the stabilizing effect of hydrogen bonding. The role of the glycine here is similar to that of the water molecules in microsolvated clusters ${ }^{12}$. Stabilization arises from the polarization of the acceptor oxygen by the glycine, i.e. $\mathrm{O}_{2}$ in $\mathrm{T}_{1,2}$-Gly and $\mathrm{T}_{2,3}$-Gly, and $\mathrm{O}_{4}$ in $\mathrm{T}_{3,4}$-Gly. Except for the latter, the total charge on the thymine is practically -1e, thus confirming that the excess electron localizes on the thymine. The situation is different for $\left[\mathrm{T}_{3,4}-\mathrm{Gly}\right]^{-}$, where $\mathrm{q}_{T}$ is -0.63e. This, however, is somewhat misleading. By inspecting the bottom panel in Fig. 2, it can be seen, highlighted by a (red) circle, that the proton $\mathrm{H}_{1}^{\prime}$ has transferred from the glycine to the thymine. Effectively, this proton now forms a complex with the thymine. If we consider the sum of Mulliken charges including $\mathrm{H}_{1}^{\prime}$, then the electronic charge of the complex is again close to -1e. Therefore, the thymine-proton complex constitutes a neutral entity.

6 | Journal Name, 2010, [vol], $1-20$ 
The values obtained for the AEA are very sensitive to the properties of the hydrogen bonds. It is known that binding to the acceptor sites increases the AEA, while binding to donor sites has the opposite effect, although in a smaller amount ${ }^{12}$. In the present case we have one acceptor and one donor site. Hence, the AEA values reported in table 1 result from a competition between these two compensating effects, together with the geometric differences in the hydrogen bonds arising from the different electronic distributions.

In order to obtain a quantitative relation between the proton transfer and the charge states of the thymine, we define the proton transfer strength as a dimensionless index: $\xi=1-2 R_{H A} \cos (\theta) / R_{D A}$, where $R_{D A}$ is the distance between the atoms of proton donor and acceptor, and $R_{H A}$ is the distance between the proton and its acceptor, $\theta$ is $\angle D A H$. When $\xi>0$, the proton transfer occurs. Larger $\xi$ means stronger transfer of the proton to the acceptor. A positive correlation between $\xi_{H_{1}^{\prime}}$ and $q_{T}$ can be found in Table 1 . In each dimer, the total charge of the thymine will be reduced more or less from -e, depending on the transfer strength of $\mathrm{H}_{1}^{\prime}$.

Protonation events are significant as they have been shown to be a competing mechanism to DNA strand breaks ${ }^{26}$. The concept of proton transfer will become important also in the condensed phase as a determination for where the excess electron will be localized.

\subsubsection{Dynamics}

To gain an understanding of the dynamics of T-Gly anion dimers, we added the excess electron with zero kinetic energy to the last configuration of a first-principles MD trajectory at the level of PBEO theory of each neutral dimer at $300 \mathrm{~K}$, and followed the dynamics for further 3 ps. The time evolution of $\sigma_{T}$, $q_{T}$ and $\xi_{H_{1}^{\prime} \rightarrow T}$ is shown in Fig. 3. In the first $20 \mathrm{fs}, \sigma_{T}$ increases from zero to one for all three dimers. After that, $\sigma_{T}$ fluctuates around 1 with a minor deviation of 0.002 . The first 20 fs see all $q_{T}$ decrease from zero to values around $-0.9 \mathrm{e}$. This picture is consistent with the one portrayed in previous work ${ }^{12}$, where the excess charge localized around the thymine in times of the order of 10-25 fs. However, each $q_{T}$ evolves differently. In $\left[\mathrm{T}_{2,3}-\mathrm{Gly}\right]^{-}, q_{T}$ stays close to $-0.93 \mathrm{e}$ excercising small fluctuations of the order of $0.05 \mathrm{e}$. A similar scenario is observed in $\left[\mathrm{T}_{3,4}-\mathrm{Gly}\right]^{-}$, where within $100 \mathrm{fs} q_{T}$ reduces from $-0.9 \mathrm{e}$ to $-0.63 \mathrm{e}$, and fluctuates around this value thereafter. Fluctuations here are somewhat larger, around 0.1e. A different situation is 


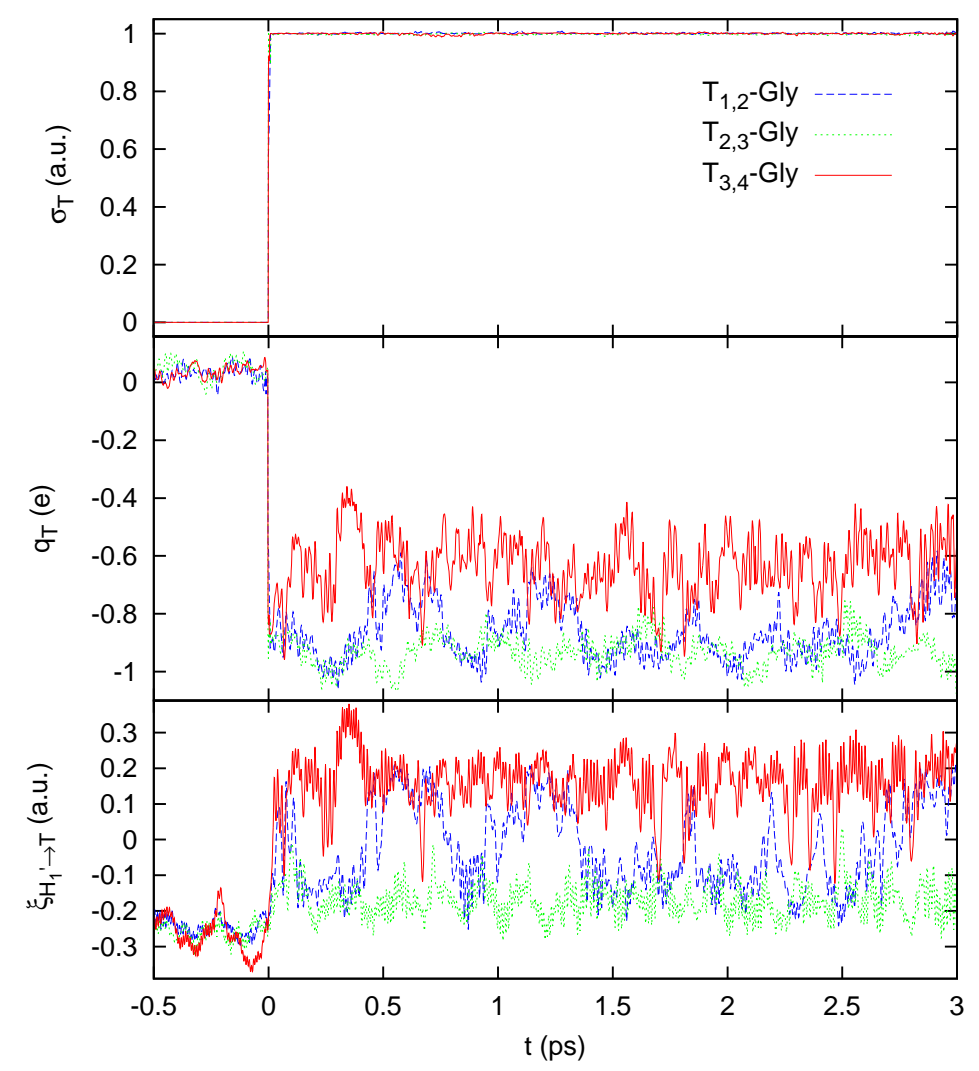

Fig. 3 (colour online) The time-evolution of the total spin density on thymine $\sigma_{T}$ (top), the sum of partial atomic charges on thymine $q_{T}$ (middle), and the transfer strength $\xi$ of $\mathrm{H}_{1}^{\prime}$ from glycine to thymine (bottom) of three dimers in ab initio MD simulations. The states before the electron attachment are shown as $t<0$.

observed in $\left[\mathrm{T}_{1,2}-\mathrm{Gly}\right]^{-}$, where $q_{T}$ oscillates between -1e and -0.6e in a seemingly bimodal way.

We can rationalize this behaviour in terms of proton transfer between the glycine and the acceptor oxygen in the thymine. In the neutral dimers all $\xi_{H_{1}^{\prime} \rightarrow T}$ are around -0.25 , meaning that there is no proton transfer. Upon attachment of the excess electron, $\xi_{H_{1}^{\prime} \rightarrow T}$ quickly increases in all three cases, but in different amounts that are correlated to the values of $q_{T}$. In $\left[\mathrm{T}_{2,3}-\mathrm{Gly}\right]^{-}, \xi_{H_{1}^{\prime} \rightarrow T}$ shifts from -0.25 to $-0.16 \pm 0.06$, meaning that the proton moves towards the center of the H-bond, but it does not transfer. This is consistent with a value of $q_{T} \approx-0.93 \mathrm{e}$. In $\left[\mathrm{T}_{3,4}-\mathrm{Gly}\right]^{-}, \xi_{H_{1}^{\prime} \rightarrow T}$ rises to 0.16 within $100 \mathrm{fs}$ and then fluctuates around this value, with a standard deviation of 0.07 . This corresponds to a quick and stable proton transfer from the glycine to the thymine, and is again consistent with the value of $q_{T} \approx-0.63 \mathrm{e}$. As in the static case, if 
we consider the thymine-proton complex, the electronic charge is close to -1e, and the complex is neutral. In the case of $\left[\mathrm{T}_{1,2}-\mathrm{Gly}\right]^{-}, \xi_{H_{1}^{\prime} \rightarrow T}$ switches periodically from -0.2 (glycine) to 0.2 (thymine), consistently with the bimodal distribution of $q_{T}$.

Therefore, for the dimers we can say that by transferring the proton to the thymine, the glycine (in general the amino acid) contributes to the neutralization of the excess electron. Since the chemical activity generally decreases with decreasing charge, the amino acid can behave as an activity quencher for the anionic nucleobase radical. As a result, the free energy barrier for electron transfer from the nucleobase to the backbone of DNA will be raised as in the case of protonation from water ${ }^{26}$, thus reducing the risk of single strand break, as shown in experiments ${ }^{27,28}$.

\subsection{Condensed phase glycine}

As a preparation for the simulations of a thymine solvated in glycine, we performed a study of pure glycine in a periodic box, which allowed us to gain an understanding of its molecular structure. In the gas phase, glycine is found in its canonical (neutral) form, while in aqueous solution it transforms to a zwitterionic form where a proton transfers from the acid to the amino group. The zwitterionic form is more stable than the nuetral by about $7 \mathrm{kcal} / \mathrm{mol}$, with an interconversion barrier around $12 \mathrm{kcal} / \mathrm{mol}^{41}$. Microsolvation studies suggest that the neutral form transforms into a zwitterion only after the addition of seven solvation waters $^{42}$. At room temperature pure glycine forms a hydrogen-bonded solid, of which three polymorphic crystal structures, $\alpha$-, $\beta$ - and $\gamma_{-}$, are known ${ }^{43}$. This polymorphism makes the molecular structure and degree of crystallinity of the sample dependent on the growth conditions, presence of additives, etc. As a matter of fact, it is perfectly possible to obtain amorphous glycine samples ${ }^{44}$.

Instead of studying a crystalline phase of glycine, we preferred to generate an amorphous sample, deeming it more representative of the physiological emvironment, although a more accurate situation would have been polyglycine, as it is more closely related to proteins. To this end, we prepared an amorphous sample of 32 canonical glycine molecules in a a cubic box of $15.05 \AA$ side, and equilibrated it for $1 \mathrm{~ns}$ via classical MD simulation using the CHARMM force field ${ }^{31,32}$. After this initial equilibration, we switched to the 
DFT simulations using the same level of theory and basis set as in the T-Gly 32 simulations (see Section 2). We first ran a 0.5 ps ab initio optimization with PBE theory to adjust geometries from CHARMM to DFT, and then we collected 15 ps of NVT dynamics at $300 \mathrm{~K}$.

The main result of this simulation is that there is an initial phase, lasting about $6 \mathrm{ps,} \mathrm{which} \mathrm{is} \mathrm{dominated}$ by proton transfer between the various glycines in the sample. After this, a dynamical equilibrium is reached where five different species can be identified based on the following bonding criterion: if the distance between the proton and an atom belonging to a molecule $\mathrm{X}$ is less than 1.2 times the equilibrium bond length $R_{0}$, then the proton is considered part of this molecule $(\mathrm{X}+\mathrm{P})$. Otherwise, it either belongs to another molecule or is considered a free proton. The four glycine-based species present in this sample are: canonical $\left(\mathrm{Gly}_{C}\right)$, zwitterionic $\left(\mathrm{Gly}_{z}\right)$, deprotonated $(\mathrm{Gly}-\mathrm{P})$, and protonated $(\mathrm{Gly}+\mathrm{P})$. In addition, there are also free protons $(\mathrm{P})$, which are transferring between glycines. It is important to remark that the distribution of molecules amongst these species depends on the bonding criterion adopted. In Fig.4 we show the timeevolution of the distribution between the various species present in the sample. It can be seen that, after equilibrium has been achieved, fluctuations in these quantities are very small, basically one unit in each direction. The 32 original molecules appear to split equally between the four glycine-based species, while there is an average of 1.6 free protons in the 32-glycine sample.

\subsection{Thymine solvated in glycine}

To study the situation of high amino acid concentration, we put the neutral dimer of Section 3.1.2 into a cubic cell of $15.05 \AA^{3}$ under periodic boundary conditions, and randomly added an additional 31 glycines. These simulations were performed only for two of the three cases above, $\mathrm{T}_{1,2}$-Gly and $\mathrm{T}_{3,4}$-Gly, as $\mathrm{T}_{2,3}$-Gly behaved similarly to the former. The corresponding solvated systems were named $\mathrm{T}_{1,2}-\mathrm{Gly}_{32}$ and $\mathrm{T}_{3,4^{-}}$ Gly $_{32}$. Before moving onto the first-principles description, these random configurations were equilibrated using classical MD with CHARMM force field. The final configurations were then used as initial configurations for the first-principles simulations. These are shown in panels (A) and (B) of Fig. 5. After switching to the first-principles decription, we re-equilibrated the systems under NVT conditions at $300 \mathrm{~K}$ with the 


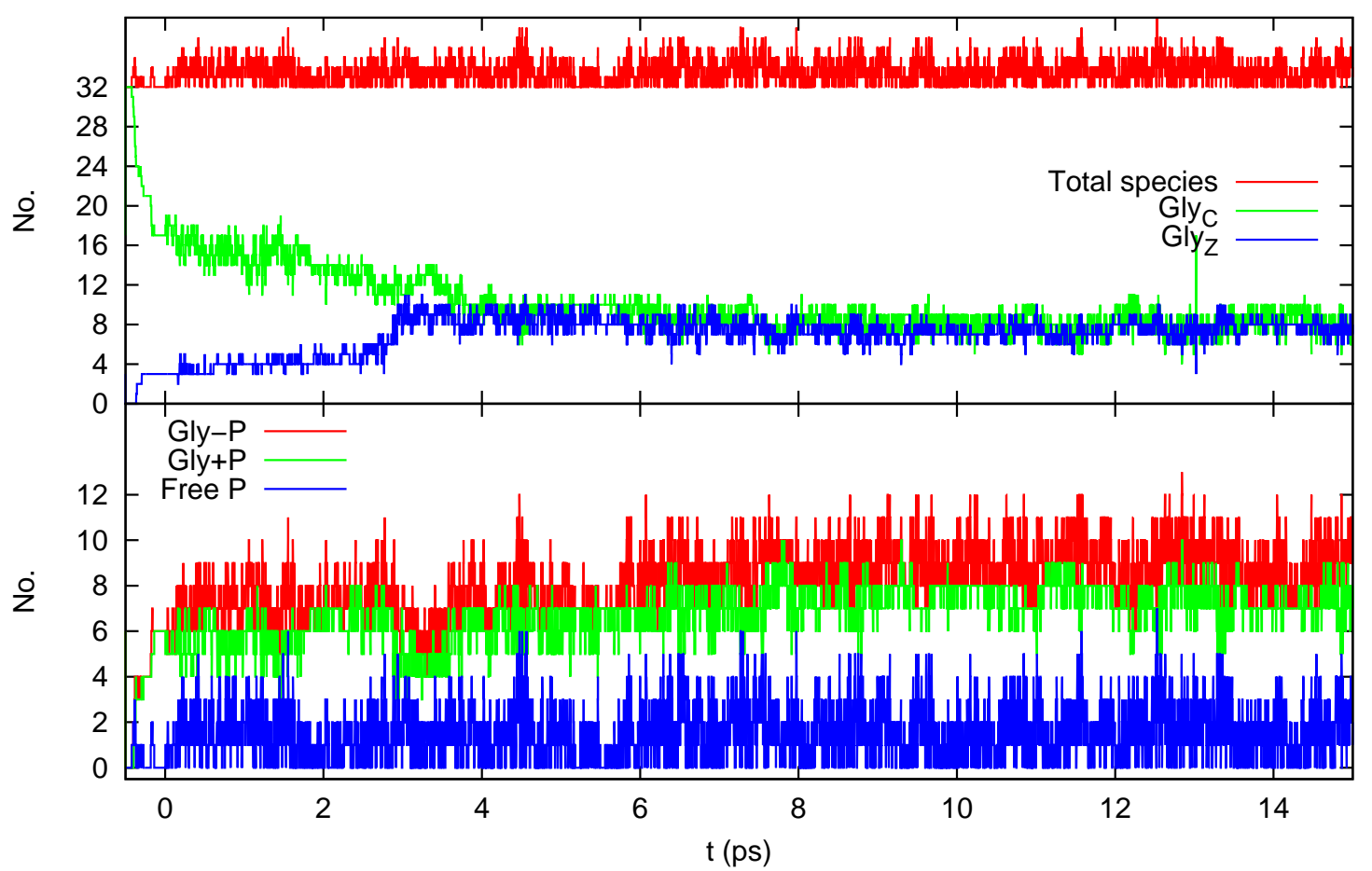

Fig. 4 (color online) Time evolution of the number of molecules of each species in a sample of amorphous glycine. A proton bonding criterion of $R<1.2 R_{0}$ has been used. The $0.5 \mathrm{ps}$ of $t<0$ corresponds to optimization, while the remainder is molecular dynamics. 


\section{DFT method at the level of PBE theory. In the equlibrations the local T-gly dimer configurations are}

maintained. Therefore, we took the last configuration of the equlibration as a representative sample. Subsequently, we added an excess electron with zero kinetic energy and continued the DFT MD simulation for further 5 ps. The configurations obtained after the first-principles thermalization are shown in Fig. $5\left(\mathrm{~A}^{\prime}\right)$ and $\left(\mathrm{B}^{\prime}\right)$.
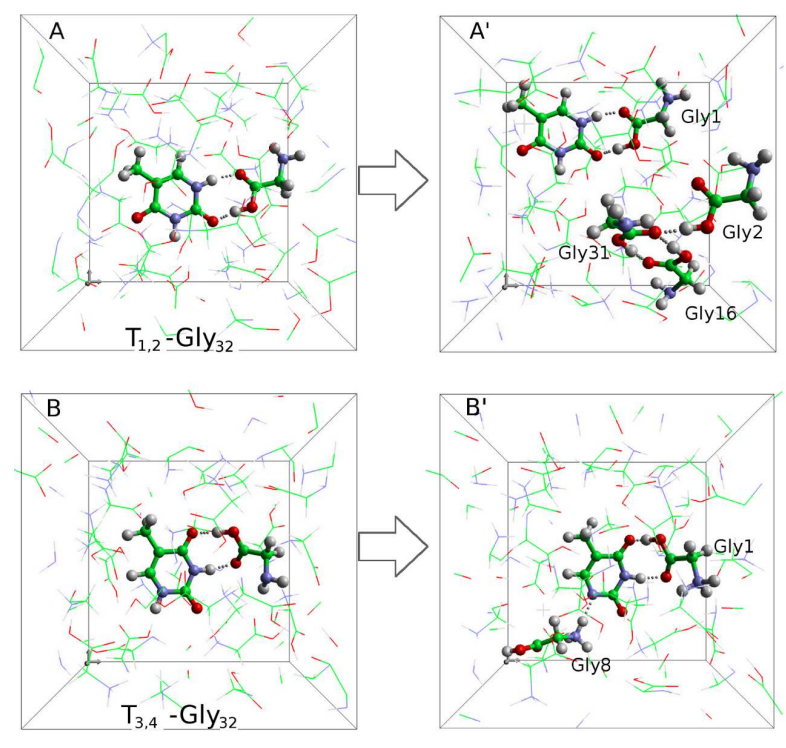

Fig. 5 (colour online) Starting configurations for the first-principles MD simulations of $T_{1,2}-G_{1} y_{32}(A)$ and $\mathrm{T}_{3,4}-\mathrm{Gly}_{32}(\mathrm{~B})$. The thermalized configurations are shown in $\left(\mathrm{A}^{\prime}\right)$ and $\left(\mathrm{B}^{\prime}\right)$. The thymine and relevant glycines are highlighted with tube and ball.

\subsection{1 $\mathbf{T}_{1,2}-\mathrm{Gly}_{32}$ : protection through physical shielding}

Fig. $5\left(\mathrm{~A}^{\prime}\right)$ reveals that the local $\mathrm{T}_{1,2}$-Gly arrangement of the dimer is preserved in the condensed phase model $\mathrm{T}_{1,2}-\mathrm{Gly}_{32}$. Snapshots of the spin density at different times are shown in Fig. 6. At the very beginning, the spin density is largely delocalized over the thymine and a few glycines. Then, as the dynamics proceeds, the molecules undergo small structural re-arrangements that facilitate the localization of the excess electron on a single molecule. In the present case, it evidently settle in one of the glycines, that we call Gly31.

As shown in Fig. 7, the total spin on Gly31, $\sigma_{G l y 31}$, increases from zero to 0.96 within $100 \mathrm{fs}$, and stays around $0.96 \pm 0.05$. In contrast, the total spin on the thymine, $\sigma_{T}$, remains always close to zero. Therefore, 


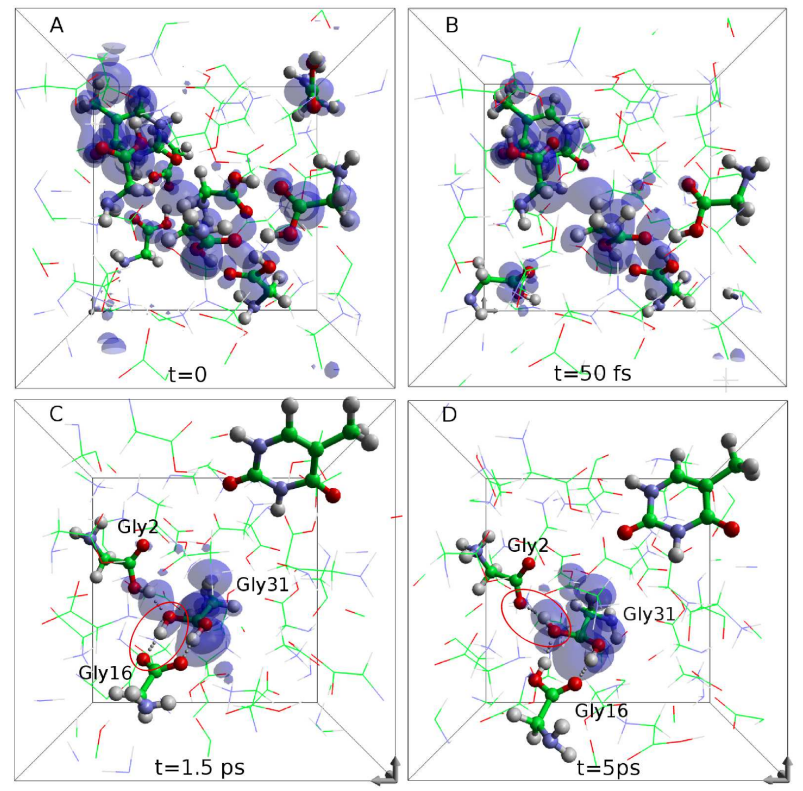

Fig. 6 (colour online) Snapshots of the spin density on $\mathrm{T}_{1,2}-\mathrm{Gly}_{32}$ during the first-principles MD simulation. The proton transfer from Gly2 and Gly16 to the thymine is highlighted in panels (C) and (D), respectively.

the Gly31 creates a physical shielding by scavenging the excess electron, leaving the thymine intact. This mechnism was not observed in the dimers.

Why is it that, in the condensed phase, there are situations when the excess electron prefers to localize on a glycine instead of the thymine? The answer can be found in the local intermolecular interactions in this configuration. As shown in $\left(\mathrm{A}^{\prime}\right)$ of Fig.5, after thermalization, Gly31 is hydrogen-bonded to Gly2 and Gly16 through two $\mathrm{H}_{1}^{\prime}$ protons. This local structure is particularly attractive for the excess electron, as proton transfer will stabilize the electron. Indeed, as shown in Fig.7, once the electron is attached, both the transfer strength variables, $\xi_{H_{1 G l y 2}^{\prime} \rightarrow G l y 31}$ and $\xi_{H_{1 G l y 16}^{\prime} \rightarrow G l y 31}$, increase as the excess electron localizes around Gly31.

In the first $600 \mathrm{fs}$, it is $\mathrm{H}_{1 G l y 2}^{\prime}$ that transfers to Gly2. At $t=600 \mathrm{fs}$, however, it switches back while $\mathrm{H}_{1 G l y 16}^{\prime}$ transfers from Gly16. At $t=2 \mathrm{ps}$, the proton switch happens again in reverse. After that, $\xi_{H_{1}^{\prime} G l y 2 \rightarrow G l y 31}$ stays around 0.24 while $\xi_{H_{1}^{\prime} G l y 16} \rightarrow$ Gly31 fluctuates around -0.24. We have stopped the simulation at $5 \mathrm{ps}$, but there is nothing to prevent this pair of protons from switching regularly in thermal equilibrium. This competition shows that one and only one proton transfer is required to stabilize the ex- 


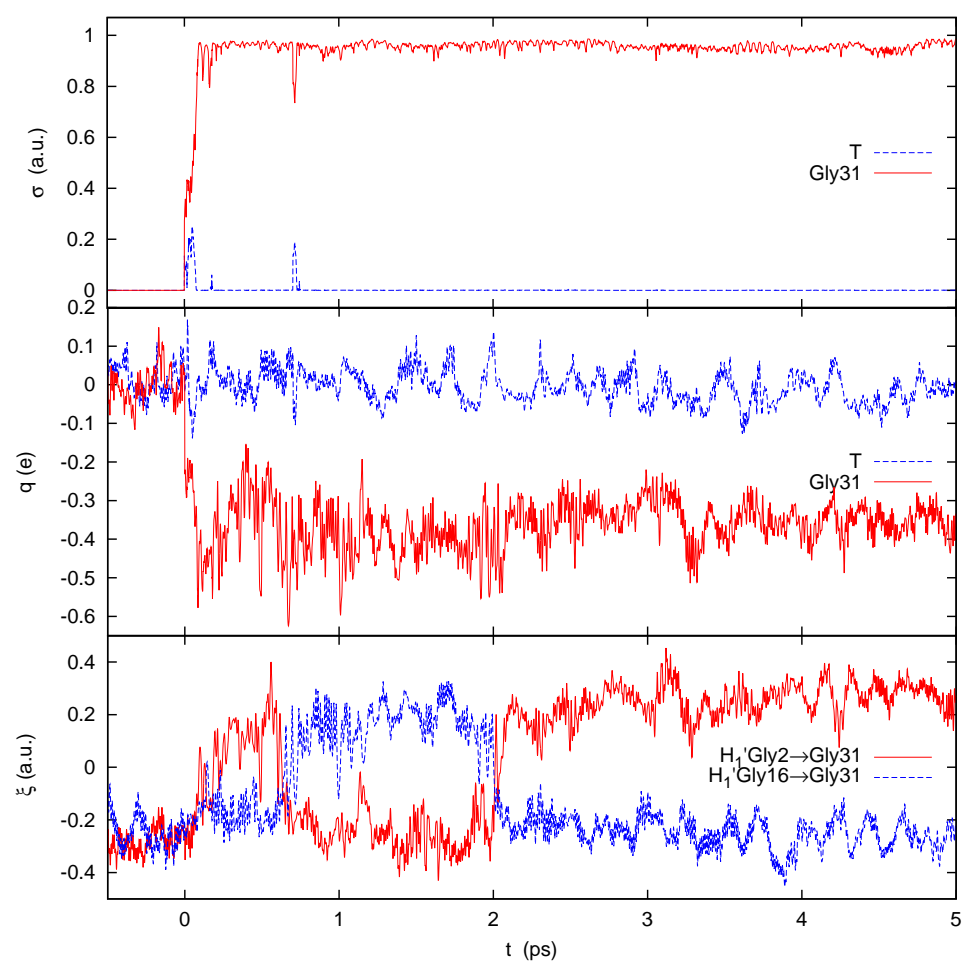

Fig. 7 (colour online) The time-evolution of the sum of spin density $\sigma$ (top) and partial charges $q$ (middle) on thymine and the 31 st glycine, and the proton transfer strength $\xi$ from the 2 nd (blue line) and 16th glycines to the 31 st glycine (bottom). The values of these parameters during the last $0.5 \mathrm{ps}$ of thermalization are shown as $t<0$.

cess electron on a glycine. If a second proton is transferred from a neighboring glycine, then another proton must be shuttled away thus maintaining charge neutrality.

As a result of electron localization, the net charge on Gly31 $\left(q_{G l y 31}\right)$ initially decreases from zero to -

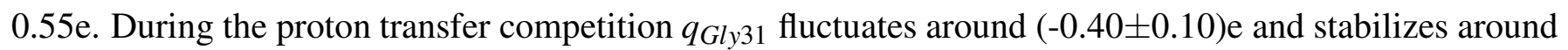
-0.35 e after 2 ps. Notice that when the transferred protons and their associated electronic basis orbitals are considered together with Gly31, the total charge of the fragment adds up practically to zero, exactly as for the dimers.

Hence, we can conclude that in the $\mathrm{T}_{1,2}-\mathrm{Gly}_{32}$ case the local structure promotes the localization of the excess electron around a glycine, which is stabilized through protonation from neighboring glycines. This occurs because, in this realization of the condensed phase, the LUMO of the solvated $\mathrm{T}_{1,2}$-Gly dimer becomes unfavorable against an empty orbital in one of the glycines in which both oxygens, $\mathrm{O}_{2}^{\prime}$ and $\mathrm{O}_{3}^{\prime}$, 
are protonated. In other words, the nucleic acid and the amino acid compete for the excess electron. Notice that the AEA of this dimer is lower than that of $\mathrm{T}_{3,4}$-Gly (see table 1).

This protection mechanism can be thought of as a physical shielding whereby a glycine scavenges the excess electron from the thymine. There is no proton transfer between the thymine and glycine in the process, with the thymine acting as a mere spectator.

\subsection{2 $\mathbf{T}_{3,4}-G^{-} y_{32}:$ protection through protonation}

The thermalized structure of $\mathrm{T}_{3,4}-\mathrm{Gly}_{32}$ is shown in Fig.5 $\left(\mathrm{B}^{\prime}\right)$. It can be seen that the $\mathrm{H}_{1}$ of the thymine has transferred to the $-\mathrm{NH}_{2}$ group of Gly8, while $\mathrm{H}_{1}^{\prime}$ in Gly11 has transfered to the $-\mathrm{NH}_{2}$ group of Gly1. Meanwhile, the hydrogen bonds between the thymine and Gly1 are maintained. As a proton donor, the net charge of the thymine $\left(q_{T}\right)$ is about $-0.45 \pm 0.05 \mathrm{e}$, while $q_{G l y 1}$ and $q_{G l y 8}$ are $0.40 \pm 0.04 \mathrm{e}$ and $0.38 \pm 0.04 \mathrm{e}$ individually as they are protonated.

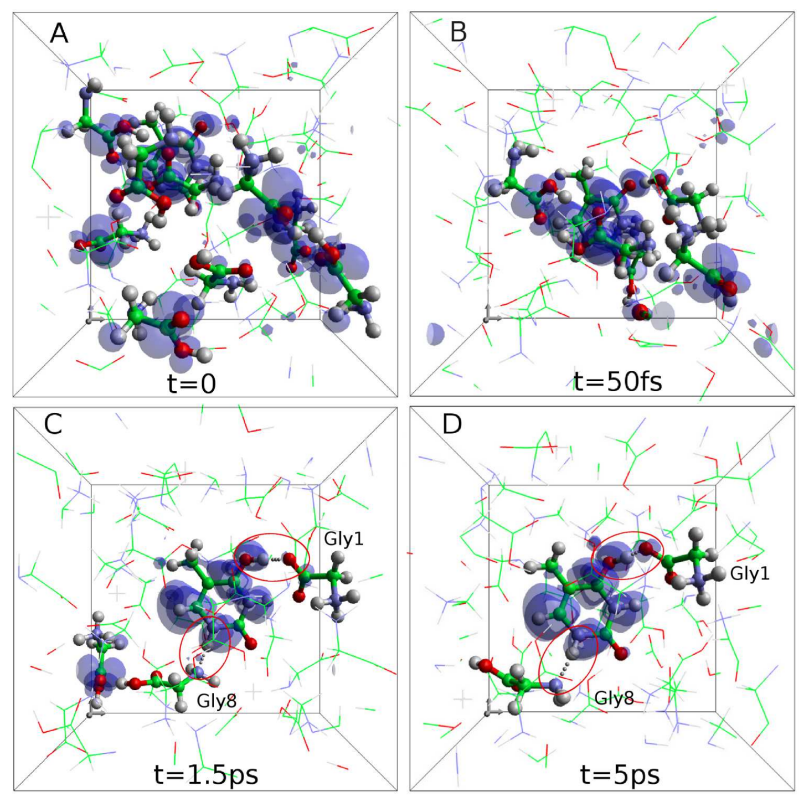

Fig. 8 (colour online) Snapshots of the spin density distribution on the $\mathrm{T}_{3,4}-\mathrm{Gly}_{32}$ system along the $a b$ inito simulation. The transfer of $\mathrm{H}_{1}$ back to the thymine from Gly8 and $\mathrm{H}_{1}^{\prime}$ from Gly1 to the thymine are highlighted with red circles in (C) and (D).

Snapshots of the spin density distribution in the $\mathrm{T}_{3,4}-\mathrm{Gly}_{32}$ system are shown in Fig.8. When the electron is vertically attached at $t=0$, the spin density is widely distributed in the system, as is in the case of $\mathrm{T}_{1,2^{-}}$ 
Gly $_{32}$. However, in this case the excess electron localizes around the thymine instead of a glycine. As shown in Fig.9, $\sigma_{T}$ increases from zero to 0.95 within $100 \mathrm{fs}$, and stays around that value from then on.

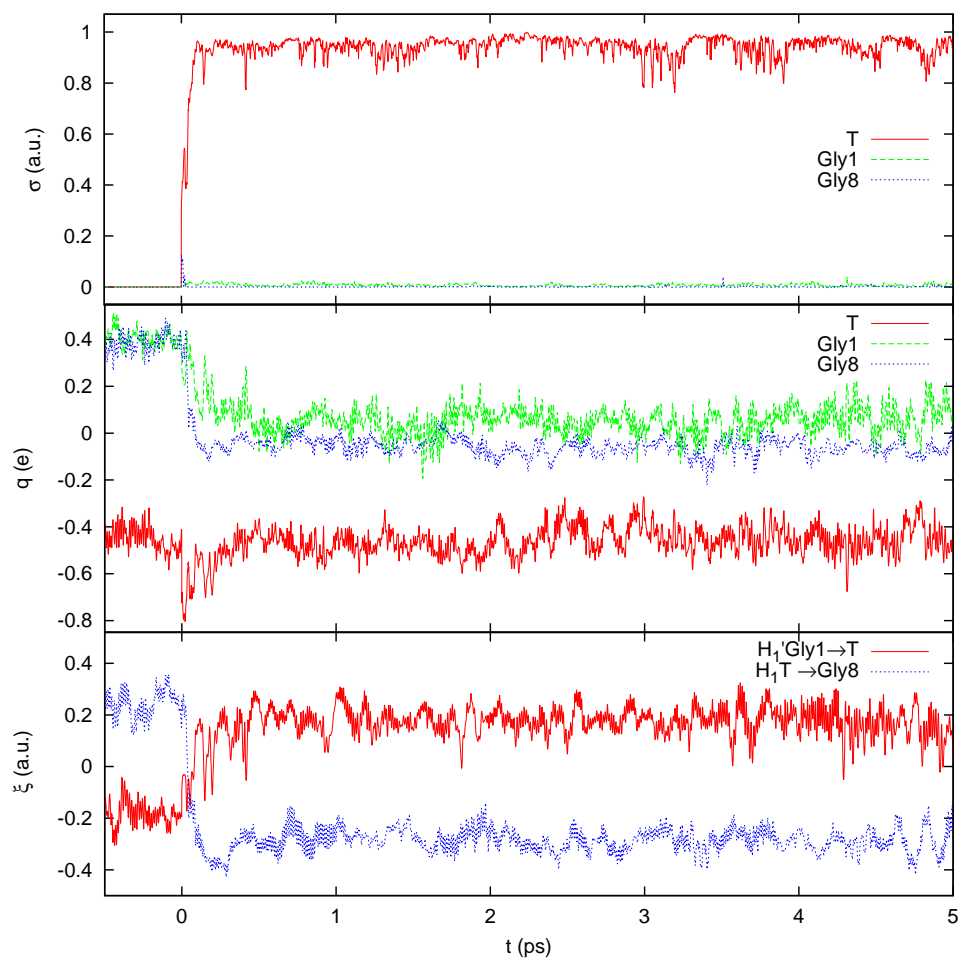

Fig. 9 Time evolution of the total spin density $\sigma$ (top), total atomic charges (middle) on the thymine $\left(q_{T}\right)$, Gly 1 $\left(q_{G l y 1}\right)$ and Gly8 $\left(q_{G l y 8}\right)$, and transfer strength $\xi$ of $\mathrm{H}_{1}$ from thymine to Gly8 and of $\mathrm{H}_{1}^{\prime}$ from Gly1 to the thymine (bottom). The quantities during the last $0.5 \mathrm{ps}$ of thermalization are shown as $t<0$.

The thymine charge $q_{T}$ decreases from -0.45 e to about $-0.80 \mathrm{e}$ in the first 40 fs after injection of the excess electron, but it quickly goes back to $-0.45 \mathrm{e}$ in the following $250 \mathrm{fs}$. This is in contrast to $q_{G l y 8}$, which decreases from $0.38 \mathrm{e}$ to zero within $200 \mathrm{fs}$, and to $q_{G l y 1}$, which also decreases from $0.40 \mathrm{e}$ to zero within 500 fs, remaining neutral for the remainder of the simulation.

The fact that $q_{T}$ returns quickly to its original value can be related to the transfer of $\mathrm{H}_{1}$ back from Gly8 and the additional proton transfer from the neighboring Gly1. This follows from the lower panel of Fig.9 where it can be seen that, after electron attachment, $\xi_{H_{1 T} \rightarrow G l y 8}$ decreases from 0.24 to -0.28 within 250 fs $\left(\mathrm{H}_{1}\right.$, which had transferred to Gly8, returns to the thymine). At the same time, $\xi_{H_{1}{ }^{\prime} G l y 1} \rightarrow T$ increases from -0.18 to 0.17 indicating that $\mathrm{H}_{1}^{\prime}$ in Gly1 also transfers to the thymine, as highlighted in the snapshots (C) 
and (D) in Fig.8. As a result of these two protonation events, $q_{T}$ reduces from $-0.8 \mathrm{e}$ to $-0.45 \mathrm{e}$ with the excess electron localized on it. Moreover, if the basis orbitals corresponding to $\mathrm{H}_{1}$ and $\mathrm{H}_{1}{ }^{\prime}$ are included in the computation of the Mulliken charges, the total charge of the protonated thymine again turns out to be practically zero $(-0.14 \pm 0.06 \mathrm{e})$. The neutralization of the anionic thymine radical due to protonation suggests a reduction of its chemical activity and a decrease of the mobility of the excess electron. In this way, pre-protonated amino acids can provide protection of DNA by acting as a source of protons that, when transferred, inhibit further reactions of the LEEs.

In physiological and experimental conditions, despite the vast variety of local configurations involving nucleic and amino acids, intermolecular interactions between them occur via hydrogen bonds. Furthermore, such hydrogen bonds between nucleic acids and the $-\mathrm{OH}$ and $-\mathrm{NH}_{3}$ groups of amino acids are universal and consitute natural proton sources ${ }^{14}$. Therefore, it can be argued that the increase of the ratio of amino acid to nucleobase above 1:1 will always promote the protection of DNA by LEEs, either chemically as shown in the case of $T_{3,4}-G_{1} y_{32}$, or physically as shown for $T_{1,2}-G_{1} y_{32}$. It should be noted that, with high ratio of amino acids, the local configuration of the T-gly dimers might change on a time-scale longer than our simulation. In this way, these two different kinds of protections will take place randomly in reality.

\section{Conclusions}

Since the discovery of LEE-induced strand breaks in DNA, a large number of experimental and theoretical results have been gathered concerning the interaction of LEEs with DNA strands and its constituents. This has been done for the gas phase, dry condensed states, and under micro-solvated conditions. Much more scarse are studies of the interaction between DNA and proteins in the presence of LEE, in particular the influence of neighboring amino acids on strand breaks, in spite of the conspicuous vicinity of histones in the physiological environment. It is, therefore, very important to include amino acids in the picture in order to understand radiation-induced DNA damage under realistic conditions.

In this paper we investigated the notion that amino acids can play a protective role to DNA in the presence of excess LEE, by studying via first-principles molecular dynamics simulations a model system 
composed of thymine and glycine. Our results show that, at room temperature, the amino acid can protect DNA in two ways: chemically and physically. Which one of the two is realized depends on the specific solvation structure of the nucleic acid, i.e. on the local configuration of glycines around the thymine. When protons can be readily transferred from the amino acid to the nucleobase, the electron initially localizes around the base inducing a very fast, barrier-less proton transfer from a neighboring amino acid. This proton serves to stabilize the excess electron in the nucleobase, by reducing the net partial charge on it (chemical mechanism). Secondly, the excess electron can be scavenged directly by the amino acid and stabilized by proton transfer from another, neighbouring amino acid, thus increasing the electron affinity of the former relative to the nucleobase. The physical shielding requires a predisposition for proton transfer to the oxygen in the carboxylic acid group of one of the amino acid molecules. Both mechanisms, chemical and physical, create obstacles for further reactions of the excess electron within a DNA strand, like electron transfer to the backbone leading to strand breaks. With increasing ratio of amino acid to nucleic acid, protection will be enhanced and DNA strand breaks by LEEs will decreased accordingly, as shown experimentally.

As it has been shown in this and other works ${ }^{26}$, proton transfer is protective against low-energy electron induced DNA damage, either directly or indirectly. It is then natural to postulate that, in a realistic cellular environment, all proton sources in the vicinity of $\mathrm{DNA}^{45}$ will be involved in its protection from the abundant secondary electrons produced in ionizing radiation events. Having confirmed the important role of protons in inhibiting some DNA damage pathways, one can now suggest the possibility of harnessing this feature by introducing therapeutic agents that release protons in damaged areas.

\section{Acknowledgements}

This work was supported the National Natural Science Foundation of China (Grant No.11105075), the Jiangsu Government Scholarship for Overseas Studies (Grant No. JS2012-105) and the Qinlan Project of Jiangsu Province (2012). The simulations were carried out in the HECToR and ARCHER facilities under the UKCP consortium allocation. We thank Nigel Mason and Gareth Tribello for fruitful discussions.

18 | Journal Name, 2010, [vol], 1-20 


\section{References}

1 B. Boudaffa, P. Cloutier, D. Hunting, M. A. Huels and L. Sanche, Science, 2000, 287, 1658-1660.

2 E. Scifoni, E. Surdutovich and A. V. Solov'yov, Phys. Rev. E, 2010, 81, 021903.

3 S. M. Pimblott and J. A. LaVerne, Radiat. Phys. Chem., 2007, 76, 1244.

4 S. Wesolowski, M. Leininger, P. Pentchev and H. S. III, J. Am. Chem. Soc., 2001, 123, 4023.

5 S. Walch, Chem. Phys. Lett., 2003, 374, 296.

6 D. Roca-Sanjuan, M. Merchan, L. Serrano-Andres and M. Rubio, J. Chem. Phys., 2008, 129, 095104.

7 J. Gu, N.-B. Wong, Y. Xie and H. S. III, J. Chem. Eur., 2010, 16, 13155.

8 J. Rak, M. Kobyecka and P. Storoniak, J. Phys. Chem. B, 2011, 115, 1911-1917.

9 S. Kim, S. Wheeler and H. S. III, J. Chem. Phys., 2006, 124, 204310.

10 S. Kim and H. Schaefer, J. Chem. Phys., 2007, 126, 064301.

11 J. Gu, Y. Xie and H. S. III, Nucleic Acids Res., 2007, 35, 5165.

12 M. Smyth and J. Kohanoff, Phys. Rev. Lett., 2011, 106, 238108.

13 S. Ptasinska and L. Sanche, Phys. Rev. E, 2007, 75, 031915.

14 B. Alberts, A. Johnson, J. Lewis, M. Raff, K. Roberts and P. Walter, Molecular Biology of the Cell, Garland Science, 2008.

15 S. Mukherjee, S. Majumdar and D. Bhattacharyya, The Journal of Physical Chemistry B, 2005, 109, $10484-10492$.

16 L. Pardo, M. Campillo, D. Bosch, N. Pastor and H. Weinstein, Biophysical Journal, 2000, 78, $1988-1996$.

17 N. M. Luscombe, R. A. Laskowski and J. M. Thornton, Nucleic acids research, 2001, 29, 2860-2874.

18 L. R. Rutledge, C. D. M. Churchill and S. D. Wetmore, The Journal of Physical Chemistry B, 2010, 114, 3355-3367.

19 K. L. Copeland, S. J. Pellock, J. R. Cox, M. L. Cafiero and G. S. Tschumper, The Journal of Physical Chemistry B, 2013, 117, 14001-14008.

20 K. A. Wilson, J. L. Kellie and S. D. Wetmore, Nucleic acids research, 2014, 42, 6726-6741.

21 M. Gutowski, I. Dabkowska, J. Rak, J. Nilles, D. Radisic and K. Bowen, Eur. Phys. J. D, 2002, $20,431$.

22 I. Dabkowska, J. Rak, M. Gutowski, J. Nilles and K. Bowen, J. Chem. Phys., 2004, 13, 6064.

23 A. Szyperska, A. Gajewicz, K. Mazurkiewicz and J. Leszczynski, Phys. Chem. Chem. Phys., 2011, $13,19499$.

24 N. C. Seeman, J. Rosenburg and A. Rich, Proc. Natl. Acad. Sci., 1976, 73, 804.

25 Y. Mandel-Gutfreund, O. Schueler and H. Margalit, J. Mol. Biol., 1995, 253, 470.

26 M. Smyth and J. Kohanoff, J. Am. Chem. Soc., 2012, 134, 9122-9125.

27 T. Solomun and T. Skalick, Chem. Phys. Lett., 2008, 453, 101-104.

28 S. Ptasinska, Z. Li, N. J. Mason and L. Sanche, Phys. Chem. Chem. Phys., 2010, 12, 9367-9372. 
29 S. S. Wesolowski, M. L. Leininger, P. N. Pentchev and H. F. Schaefer, J. Am. Chem. Soc., 2001, 123, $4023-4028$.

30 T. G. A. Youngs, J. Comput. Chem., 2010, 639-648.

31 A. D. Mackerell, M. Feig and C. L. Brooks, J. Comput. Chem., 2004, 25, 1400-1415.

32 A. D. MacKerell, N. Banavali and N. Foloppe, Biopolymers, 2000, 56, 257-265.

33 W. Smith, C. Yong and P. Rodger, Mol. Sim., 2002, 28, 385-471.

34 J. VandeVondele, M. Krack, F. Mohamed, M. Parrinello, T. Chassaing and J. Hutter, Comput. Phys. Commun., 2005, 167, 103-128.

35 G. Lippert, J. Hutter and M. Parrinello, Theor. Chem. Acc., 1999, 103, 124-140.

36 G. J. Martyna and M. E. Tuckerman, J. Chem. Phys, 1999, 110, 2810-2821.

37 C. Adamo and V. Barone, J. Chem. Phys, 1999, 110, 6158-6170.

38 J. P. Perdew, K. Burke and M. Ernzerhof, Phys. Rev. Lett., 1996, 77, 3865-3868.

39 S. Goedecker, M. Teter and J. Hutter, Phys. Rev. B, 1996, 54, 1703-1710.

40 R. S. Mulliken, J. Chem. Phys., 1955, 23, 1833-1840.

41 K. Leung and S. B. Rempe, J. Chem. Phys., 2005, 122, 184506.

42 S. M. Bachrach, J. Phys. Chem. A, 2008, 112, 3722-3730.

43 Y. Iitaka, Acta Crystallographica, 1960, 13, 35-45.

44 S. J. Bai, M. Rani, R. Suryanarayanan, J. F. Carpenter, R. Nayar and M. C. Manning, Journal of Pharmaceutical Sciences, 2004, 93, 2439-2447.

45 A. Kumar and M. D. Sevilla, Chem. Rev., 2010, 110, 7002-7023. 